\section{REFRACTORY MATERIALS.}

A GENERAL discussion on refractory materials was held on November 8 at the Faraday Society he chair being occupied by the president, Sir Robert Iadfield. Numerous exhibits were on view, including british, Colonial, and Indian raw materials, manufactured products, appliances, etc.

In his introductory address the president surveyed the whole range of refractory materials, and mentioned that when Belgian sand was no longer available for open-hearth furnace bottoms, those concerned were not long in discovering British sands which give practically the same results. This point was emphasised later by Mr. Cosmo Johns and Dr. Boswell.

Dr. J. W. Mellor (Stoke-on-Trent) opened the discussion with a paper on

\section{"The Texture of Firebricks."}

Dr. Mellor classed texture and refractoriness as the two most important properties of a firebrick. Numerous samples illustrating texture were exhibited, prepared by cutting across with a saw, polishing the cut face, and cementing a glass plate on with hot Canada balsam. This method of showing a brick's texture (now used, it is believed, for the first time) was suggested by Mr. Lomax.

\section{Size of Grain.}

The softening of clay material takes place gradually. From the fact that increased fineness of grain has been found to increase the contraction of fired clay bodies, presumably owing to increased surface reaction in promoting vitrification, it might be expected that pressure would also lower the softening temperature of a clay by increasing the area of contact, and this has recently been shown to be the case. ${ }^{1}$

This effect of fineness ot grain comes out prominently in the case of high-temperature fluxes, like mixtures of clay and fine-grained quartz, where the vitrifi. cation temperature may be brought so low as to spoil the firebrick. Conversely, the presence of coarsegrained quartz appreciably increases the refractoriness of fireclays; the quartz grains, however, should be angular, not rounded, the rounded quartz grains being only loosely held by the clay bind, besides which angular particles pack together more closely and form a more compact skeleton for the brick, as in samples exhibited.

\section{After-Contraction and After-Expansion.}

The firebrick manufacturer arrests the chemical reaction at a certain stage. When the brick in use is strongly heated, the uncompleted reaction is continued, giving rise to after-contraction. This after-contraction a few years ago amounted to 2 or 3 per cent., but was reduced by improved methods to I per cent., and then to $\frac{1}{2}$ per cent. It is usually impracticable to eliminate all the after-contraction in the original burning of the firebrick. Silica firebricks show an after-expansion, which may reach up to 16 per cent. The quartzose silica of clays behaves similarly, unless it be dissolved by the fluxes. The fluxes in a clay also expand about 6 per cent. in firing, so that the apparent contraction of a firebrick is a joint effect. In silica bricks the resultant effect is an expansion.

\section{Refractoriness and other Qualities.}

It is clear that conditions which increase the extent of surface of the clay particles in contact or which produce closer contact make the clay soften at a lower temperature, and this indicates how to obtain maximum refractoriness for normal conditions with a given

${ }^{1}$ J. W. Mellor and B. J. Moore, Trans. Eng. Cer. Soc., xv., II7, Igr6. NO. 2458 , VOL. 98$]$ clay. The refractoriness can be further augmented by addition of some of the higher refractories, like shrunk bauxite, shrunk zirconia, or carborundum. It should, however, be borne in mind that a coarse-grained refractory material has low crushing strength and tenacity, and is also very friable and liable to disintegration by shocks or abrasion, besides being easily penetrated by flue dusts and slags. When maximum refractoriness is not really wanted, some refractoriness may be sacrificed advantageously to improve other desirable qualities.

\section{Hand-made v. Machine-made Firebricks.}

With reference to the making of firebricks by hand or by machinery, the machines often get blamed for faults which have no direct connection with the use of the machines, but arise from differences in the method of preparing the clay, which affect the uniformity in texture of the product.

\section{Corrosion of Firebricks.}

The joints between firebricks are the weakest places in a structure. Where slags are concerned the firebricks should be fine-grained, and the jointing clay should quickly vitrify without cracking and weld the bricks together when the furnace is fired for the first time. Where bricks are exposed to corrosive vapours the chemical composition is of special importance, as well as the texture of the bricks.

Prof. T. Turner (Birmingham), referring to $\mathrm{Dr}$. Mellor's statement that angular particles give closer packing than round particles, contended that the closest packing of all is obtained by adjusting two sizes of round particles so that the smaller grains shall approximately fill up the interspaces between the larger grains.

\section{Other Refractory Materials.}

Dr. Hutton directed attention to the great value as refractories of completely shrunk silica, alumina, and magnesia, all of which can stand sudden heating and cooling.

Dr. W. Rosenhain stated that zirconia has a promising future as a refractory material, especially the purified substance. It withstands very high temperatures, but should be previously heated to a higher temperature than that to which it is to be exposed, in order to avoid cracking. It is also very apt to form a carbide in a reducing atmosphere, and the properties of the carbide are very different from those of the oxide.

J. A. A.

\section{UNIVERSITY AND EDUCATIONAL INTELLIGENCE.}

Liverpool.-The University has just received two valuable gifts-80ool. from Mr. C. Sydney Jones, to endow the chair of classical archæology, hitherto maintained by temporary guarantees, and io,oool. from Prof. and Mrs. Herdman, to establish a chair of geology. Both chairs are memorials: the first of a father, Mr. Charles W. Jones; the second of a son, Lieut. George A. Herdman, a young student, not merely of promise, but of distinction, who was killed in action in France a few months ago. Each satisfies a real need, for at Liverpool the course of study in Latin and in Greek recognises that without some knowledge of life and custom it is impossible to understand ancient history or to appreciate classical literature. The professor of classical archæology, therefore, takes an active part throughout the classical course, though his work is so arranged as to leave one term in the session free for research at home or abroad. 